\title{
GASTERÓPODOS DULCEACUÍCOLAS DE PROVIDENCIA, ARCHIPIÉLAGO DE SAN ANDRÉS, PROVIDENCIA Y SANTA CATALINA, COLOMBIA
}

\author{
Daniela CORTÉS-GUZMÁN ${ }^{1} \&$ Edgar L. LINARES ${ }^{2}$
}

\author{
${ }^{1}$ Departamento de Biología, Facultad de Ciencias, Universidad Nacional de Colombia, Sede Bogotá, \\ <dacortesgu@unal.edu.co> \\ ${ }^{2}$ Instituto de Ciencias Naturales, Facultad de Ciencias, Universidad Nacional de Colombia, Sede Bogotá, Apartado \\ 7495,<ellinaresc@unal.edu.co> \\ Recibido: 11/08/2015; aceptado: 09/12/2015
}

Cortés-Guzmán, D. \& Linares, E. L. 2016. Gasterópodos dulceacuícolas de Providencia, archipiélago de San Andrés, Providencia y Santa Catalina, Colombia. Acta Zoológica Mexicana (n. s.), 32(1): 101-110.

RESUMEN. El presente estudio describe la composición de la comunidad de moluscos en varias quebradas de Providencia, y la dinámica de éstas en tres periodos hidrológicos. Se realizaron tres muestreos en cinco quebradas de la isla, donde se recolectaron los moluscos. Se calcularon índices y descriptores de la comunidad, se describió la dinámica de las especies con la estacionalidad de la precipitación de la isla y se establecieron las preferencias de microhábitats. Se encontraron cinco especies de moluscos dulceacuícolas, de las cuales cuatro no se habían reportado para la isla ni para el archipiélago. La dinámica estacional de la comunidad de moluscos principalmente dulceacuícolas mostró un aumento poblacional en todas las especies, excepto una, durante el periodo de lluvias, y un aumento en los valores de equidad. Los microhábitats cantos rodados y raíces, fueron los más notables, y se encontró que en los sitios con mayor número de microhábitats disponibles hay mayor abundancia de moluscos.

Palabras clave: moluscos dulceacuícolas, estacionalidad, dinámica estacional.

\section{INTRODUCCIÓN}

El archipiélago de San Andrés, Providencia y Santa Catalina es un departamento perteneciente a la región Caribe colombiana, enclavado en el suroccidente del mar Caribe, localizado entre los meridianos $78^{\circ}$ y $82^{\circ}$ de latitud oeste y entre los paralelos $12^{\circ}$ y $16^{\circ}$ de longitud norte, a una distancia aproximada de $750 \mathrm{~km}$ de Cartagena de Indias, la ciudad continental colombiana más próxima, con una superficie insular total de $52.5 \mathrm{~km}^{2}$, de los que $17.2 \mathrm{~km}^{2}$ corresponden a Providencia. Limita por el oriente con islas de las Grandes y Pequeñas Antillas, por el norte con Jamaica y por el noroccidente, occidente y sur con los estados continentales de Honduras, Nicaragua, Costa Rica, Panamá y el territorio continental colombiano (Aguilera 2010).
Cortés-Guzmán, D. \& Linares, E. L. 2016. Freshwater molluscs of Old Providence, San Andrés, Providencia and Santa Catalina Archipielago, Colombia. Acta Zoológica Mexicana (n. s.), 32(1): 101-110.

ABSTRACT. The present study describes the composition of the mollusk community in various streams of Providence, and the dynamic of these during three hydrological periods. Three sampling were carried in five streams of the island, where the mollusk were collected. Indexes and community descriptors were calculated, the species dynamic with the seasonality were described and microhabitat preferences were established. Five species of freshwater mollusk, four of which were not reported for the island before, were found. The seasonal dynamic of community showed a population increase in all species, except for one, during the rainy season, and an increase in equity values. Boulders and roots microhabitats were the most remarkable, and it was found that sites with the greatest number of available microhabitats have more mollusk abundance.

Key words: freshwater molluscs, seasonality, seasonal dynamics.

La isla de Providencia, en particular, que tiene $7 \mathrm{~km}$ de largo y hasta $4 \mathrm{~km}$ de ancho, se caracteriza por un relieve quebrado con una serranía que la conforman tres ramales principales en sentido este-oeste, con alturas máximas de $350 \mathrm{~m}$, y es la única del archipiélago que tiene pequeños arroyos (IGAC 2008, Gómez et al. 2012). La isla presenta muchos arroyos que no desembocan en el mar sino que se infiltran en las depresiones costeras descargando sedimentos en la superficie terrestre: los arroyos de la vertiente occidental son de mayor longitud, persistencia y caudal que los de la vertiente oriental, en vertientes más húmedas y con coberturas vegetales de mayor extensión, mientras que los arroyos de la vertiente oriental, por su exposición directa al flujo de los vientos del este y la menor extensión en cuanto a la longitud de los cauces, presentan microcuencas con cobertura vegetal protecto- 
ra pequeña y fragmentada (POT 2000, en Gómez et al. 2012).

En las islas del Caribe, el conocimiento de los moluscos dulceacuícolas ha estado relacionado con estudios ecológicos, principalmente. La mayor parte se han relacionado con las Antillas Menores (Granada, Ferguson \& Buckmire 1974; San Vicente, Harrison \& Rankin 1976, 1978; Trinidad y Tobago, Bacon 1978, Alkins-Koo 1989, Bass 2003; Dominica y Martinica, Starmühller \& Therezien 1982; San Vicente, Santa Lucia y Granada, Harrison 1984; San Cristobal y Nieves, Bass 2006; Dominica, Bass 2007; Saba, Bass 2008; Islas Caimán, Bass 2009; y San Vicente y Las Granadinas, Bass \& de Silva 2010), unos muy pocos en las Antillas Mayores (Puerto Rico, van der Schalie 1948, Pyron \& Covich 2003, Blanco-Libreros \& Arroyave-Rincón 2009; Cuba, Vázquez et al. 2010; Jamaica, Gómez et al. 2011, Hyslop \& Hunte-Brown 2012;) y estudios ocasionales en islas de la plataforma continental (Aruba, Bonaire y Curazao, Baker 1924; CozumelMéxico, Richards 1937; Margarita-Venezuela, Richards \& Hummelinck 1940).

Para el Archipiélago de San Andrés, Providencia y Santa Catalina se han registrado 35 especies de moluscos terrestres: 24 en Providencia (cuatro dulceacuícolas), 19 en San Andrés (siete de ellas endémicas para la isla) y 2 en Santa Catalina (Pilsbry 1930a, 1930b, Wurtz 1951, Linares \& Vera 2012). Adicionalmente, el estudio de Wege et al. (2011) señala la región de las islas del Caribe como un hotspot de diversidad y endemismos para varios grupos de vertebrados y plantas, y para otros grupos de invertebrados, como los moluscos, el caso podría ser el mismo. Este es el primer estudio sobre la biología de los moluscos acuáticos del Archipiélago, a través del que se proporciona información sobre la riqueza de gasterópodos dulceacuícolas presentes en varios caños de Providencia, se describe la dinámica de poblamiento de los caños en tres periodos hidrológicos y se determina la abundancia relativa para cada una de las especies.

\section{MATERIAL Y MÉTODOS}

Se realizaron tres muestreos en tres épocas del año: el primero en el mes de julio de 2013, correspondiente a la transición entre la época seca y la lluviosa, el segundo en octubre de 2013, periodo de máxima pluviosidad, y el último en enero de 2014, correspondiente al inicio de la época seca. En cada una de estas épocas se visita- ron cinco quebradas de la isla: Lazy Hill o San Felipe, Bailey Spring, El Pico, Old Town Spring y Fresh Water (Figura 1).

Lazy Hill es una quebrada de tipo intermitente, la vegetación circundante sombrea aproximadamente el $90 \%$ del canal. En el primer muestreo el sistema estaba compuesto por por pozos aislados, que recuperaron su conectividad con el flujo de agua generado por las lluvias del segundo periodo, y se mantuvieron así hasta el tercer muestreo. Bailey Spring es un sistema que no se ve afectado por la época hidrológica debido a que está aislada del cauce de una quebrada por estructuras de captación que la rodean, y se mantuvo igual en sus características físicas durante los muestreos. El Pico es una quebrada intermitente, con abundante vegetación nativa en su parte alta, y con un paisaje intervenido por pequeños cultivos en la parte baja. En Old Town Spring hay dos cisternas de captación de agua que interrumpen el flujo de agua de la quebrada, por lo tanto no se observación cambios físicos evidentes entre los periodos de muestreo, los cuales se realizaron aguas arriba de las cisternas. Fresh Water tiene el caudal controlado por la represa ubicada aguas arriba y muestra una fuerte intervención, por lo cual no se observaron cambios físicos durante los muestreos.

En cada punto de muestreo se identificaron los microhábitats disponibles de acuerdo al sustrato predominante ( $\geq 80 \%$ de cobertura), y se clasificaron en rocas (diámetro $\geq 20 \mathrm{~cm})$, cantos rodados $(<20 \mathrm{~cm})$, hojarasca, raíces, musgos, superficie del agua y limo (Merrit et al. 1996; Wantzen \& Rueda-Delgado. 2009). Allí se colectaron los organismos utilizando una red surber de $144 \mathrm{~cm}^{2}$, con tres réplicas por sustrato siguiendo a Merrit et al. (1996) y Rueda-Delgado (2002). Se incluyeron en el muestro organismos muertos (conchas vacías). Las muestras se preservaron en etanol al 96\% y los organismos fueron depositados en las Colecciones Zoológicas (ICN), del Instituto de Ciencias Naturales de la Universidad Nacional de Colombia.

Las especies fueron determinadas con base en el estudio de los caracteres morfológicos de la concha (forma, longitud y forma de la espira, abertura, forma del opérculo, forma y tamaño del ombligo, escultura de la concha), empleando a Pilsbry (1920), Harrison (1984), Lanzer (1996), Paraense \& Pointier (2003), Vásquez et al. (2010) y Nava et al. (2011). Se calcularon índices de diversidad alfa (riqueza específica) y de equidad (índice de Simpson invertido) como indicadores de la estructura de la comunidad. 


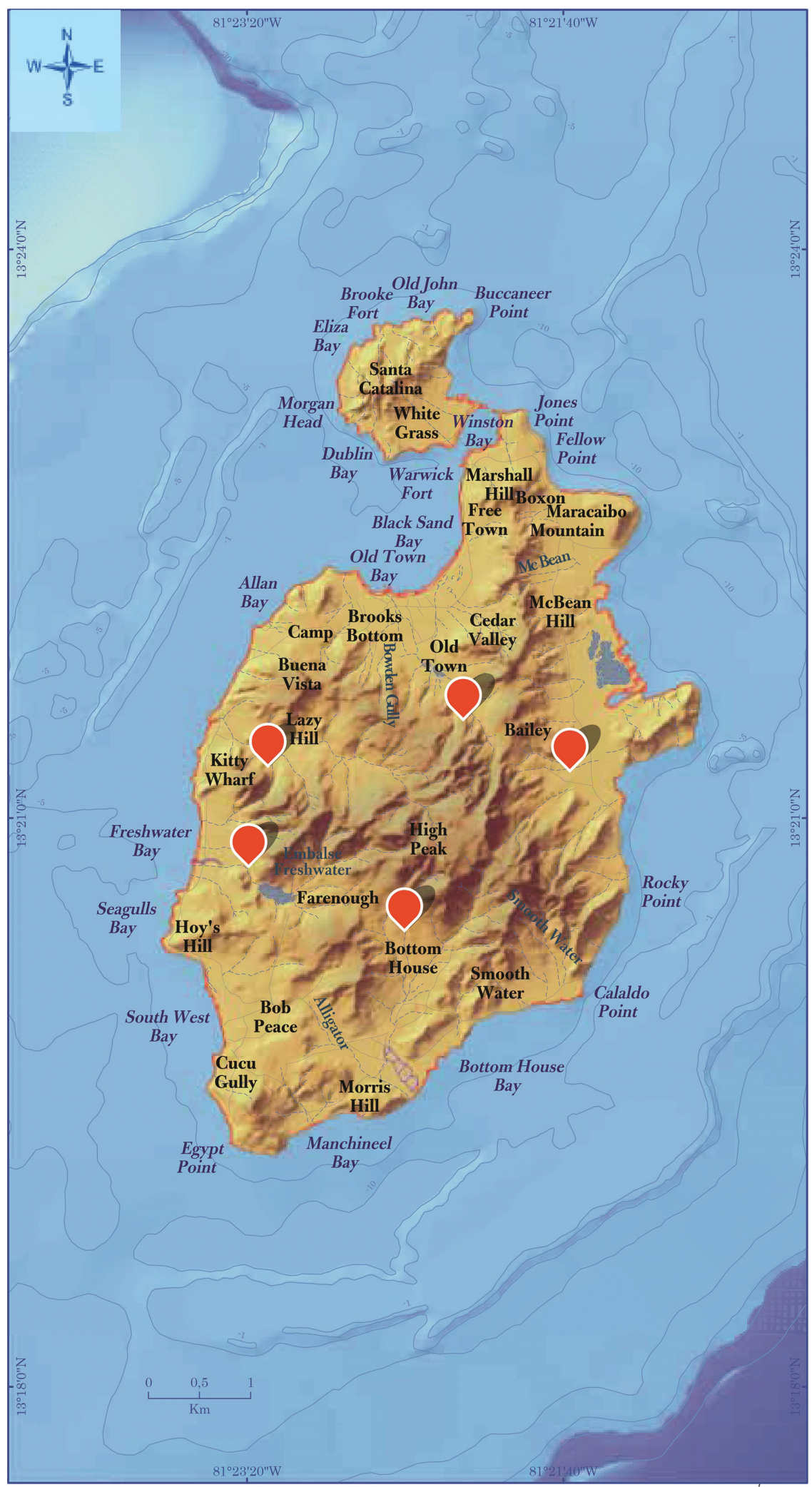

Figura 1. Mapa de Providencia y Santa Catalina, en el que se indican los sitios de muestreo en Providencia. Tomado y adaptado de Gómez et al. 2012. Atlas de la Reserva de la Biósfera Seaflower. 


\section{RESULTADOS}

En total se encontraron 4815 individuos de seis especies de gasterópodos en Providencia, pertenecientes a cinco géneros y cinco familias: Biomphalaria pallida (Adams, 1846), Planorbidae; Gundlachia radiata (Guilding, 1828), Ancylidae; Haitia acuta (Draparnaud, 1805), Physidae; Neritina punctulata Lamarck, 1816, Neritidae y Pyrgophorus coronatum (F.G. Thompson, 1968), Hydrobiidae (Cuadro 1).

La dinámica de las abundancias muestra que las especies encontradas fueron más abundantes en la época de lluvias, correspondiente al muestreo 2 , excepto para $P$. coronatum que fue más abundante en la época 1 (inicio de lluvias). Una especie ( $B$. pallida) sólo se encontró a partir del segundo muestreo. Las demás estuvieron presentes durante las tres épocas: G. radiata y $H$. acuta fueron más abundantes en la época de inicio de lluvias (1) que en la finalización de las lluvias (3), y N. punctulata fue prácticamente igual en estas dos épocas, mostrando un cambio importante sólo en la época de lluvias.

Los descriptores e índices de los sitios (Cuadro 2) muestran un aumento en la riqueza, en la abundancia (excepto en Bailey y Fresh Water) y en el índice de Simpson (excepto en Old Town) en la época de lluvias. En general, Lazy Hill tuvo la mayor riqueza, pues fue el único sitio donde se encontró $N$. punctulata, y el mayor valor en el índice de Simpson, mientras que la mayor abundancia estuvo en Bailey, principalmente por el aporte de $P$. coronatum. La menor riqueza estuvo en Fresh Water, la menor abundancia en El Pico, y el menor valor en el índice de Simpson en Bailey. En general, Bailey es el sitio con mayor dominancia de una especie, mientras que en Lazy Hill las especies se encontraron distribuidas de forma más equitativa. También la época lluviosa mostró en general para todos los sitios, una mayor equidad en las abundancias de las especies, como indicador de mayor diversidad.

En cuanto a las preferencias de microhábitat de los organismos, el sustrato de cantos rodados obtuvo el mayor número de especies asociadas (5), y el sustrato raíces obtuvo el mayor número de individuos (2332). Cabe mencionar que no todos los sustratos se encontraban disponibles en las tres épocas o en los cinco sitios de muestreo. Por ejemplo, el microhábitat de raíces sólo se encontraba en el manantial de Bailey, y el gran número de individuos se debe a $P$. coronatum. En esta misma medida, el manantial de Bailey y el sitio de Old Town obtuvieron el mayor número de sustratos disponibles (6), y así también fueron los que obtuvieron la mayor abundancia de individuos. Lazy Hill tuvo cinco sustratos disponibles y la mayor diversidad de especies, pues allí se encontraron las cinco reportadas para todo el estudio (Cuadro 2).

El microhábitat de musgo sólo obtuvo una especie, $P$. coronatum, con una abundancia significativamente baja en comparación con las abundancias de esta especie en los demás sustratos donde estuvo presente. H. acuta y $P$. coronatum fueron las especies que se encontraron en el mayor número de sustratos, $H$. acuta ausente sólo en musgo, y representaron el $4 \%$ y el $85 \%$ de la abundancia. $G$. radiata, que fue una especie con alta abundancia (7\%), sólo estuvo presente en tres de siete sustratos, con la mayoría de sus individuos en la hojarasca.

Cuadro 1. Lista de gasterópodos presentes en los caños de Providencia, incluyendo sitios de colección, período climático, número de organismos y el sustrato donde se encontró la mayor abundancia de cada especie. Época 1: transición sequía-lluvias, época 2: máxima pluviosidad, época 3: transición lluvias-sequía.

\begin{tabular}{|c|c|c|c|c|c|}
\hline Especie & Sitio & Época 1 & Época 2 & Época 3 & $\begin{array}{l}\text { Sustrato con mayor } \\
\text { abundancia }\end{array}$ \\
\hline Biomphalaria pallida & $\begin{array}{l}\text { Lazy Hill, Bailey Spring, El Pico, Old } \\
\text { Town Spring }\end{array}$ & 0 & 139 & 26 & Limo \\
\hline Gundlachia radiata & $\begin{array}{l}\text { Lazy Hill, Bailey Spring, Fresh Water, El } \\
\text { Pico }\end{array}$ & 23 & 284 & 14 & Hojarasca \\
\hline Haitia acuta & $\begin{array}{l}\text { Lazy Hill, Bailey Spring, El Pico, Old } \\
\text { Town Spring }\end{array}$ & 60 & 116 & 17 & Cantos rodados \\
\hline Neritina punctulata & Lazy Hill & 5 & 25 & 6 & Cantos rodados \\
\hline Pyrgophorus coronatus & $\begin{array}{l}\text { Lazy Hill, Bailey Spring, Fresh Water, El } \\
\text { Pico, Old Town Spring }\end{array}$ & 2205 & 1550 & 345 & Raíces \\
\hline Total individuos & & 2293 & 2114 & 408 & \\
\hline
\end{tabular}


Cuadro 2. Índices de diversidad alfa (R: riqueza específica, N: abundancia, Cinv: Índice de Simpson invertido) calculados para cada sitio en cada época de muestreo. Época 1: transición sequía-lluvias, época 2: máxima pluviosidad, época 3: transición lluvias-sequía.

\begin{abstract}
Sitio/Época
\end{abstract}

$\begin{array}{lll} & 1 & 2 \\ \text { Lazy Hill } & 4 & 5 \\ \text { Bailey } & 2 & 4 \\ \text { Fresh Water } & 2 & 2 \\ \text { El Pico } & 2 & 4 \\ \text { Old Town } & 2 & 3\end{array}$

\section{Colecciones estudiadas}

Ancylidae

\section{Gundlachia radiata (Guilding, 1828)}

Archipiélago de San Andrés, Providencia y Santa Catalina: Providencia, Fresh Water, 1320'45.6' N, 81 ${ }^{\circ} 23^{\prime} 21.18^{\prime}$ ', O, entre hojarasca del pozo 1, 40 m, 27 Ene 2014, Daniela Cortés Guzmán (Colecciones zoológicas-ICN 068064); Fresh Water, $13^{\circ} 20^{\prime} 45.6^{\prime \prime} \mathrm{N}, 81^{\circ} 23^{\prime} 21.18^{\prime}$ ' O, entre hojarasca del pozo alto, $40 \mathrm{~m}, 17$ Oct 2013, Daniela Cortés Guzmán (Colecciones zoológicas-ICN 068065); Lazy Hill-San Felipe, 1321'21.36" N, 81²3'17.52', O, entre hojarasca de la parte alta de la quebrada, $31 \mathrm{~m}, 16$ Oct 2013, Daniela Cortés Guzmán (Colecciones zoológicasICN 068066); ); Lazy Hill-San Felipe, 13²1'21.36" N, $81^{\circ} 23^{\prime} 17.52^{\prime}$ ' $\mathrm{O}$, entre cantos rodados de la parte alta de la quebrada, 31 m, 16 Oct 2013, Daniela Cortés Guzmán (Colecciones zoológicas-ICN 068067); Lazy Hill-San Felipe, $13^{\circ} 21^{\prime} 21.36^{\prime \prime} \mathrm{N}, 81^{\circ} 23^{\prime} 17.52^{\prime}$ ' O, entre hojarasca de la parte alta de la quebrada, $31 \mathrm{~m}, 27$ Ene 2014, Daniela Cortés Guzmán (Colecciones zoológicas-ICN 068068); Lazy Hill-San Felipe, 13²1'21.36" N, 81²3'17.52', O, entre hojarasca del manantial, 31 m, 27 Ene 2014, Daniela Cortés Guzmán (Colecciones zoológicas-ICN 068069); El Pico, $13^{\circ} 20^{\prime} 36.12^{\prime \prime} \mathrm{N}, 81^{\circ} 22^{\prime} 7.26 \mathrm{O}$, entre rocas de la parte alta de la quebrada, 161 m, 17 Oct 2013, Daniela Cortés Guzmán (Colecciones zoológicas-ICN 068070); El Pico, $13^{\circ} 20^{\prime} 2.7^{\prime}$ ' N, 81 $22^{\circ}$ '9.54' O, entre cantos rodados de la parte baja de la quebrada, 112 m, 29 Ene 2014, Daniela Cortés Guzmán (Colecciones zoológicas-ICN 068071).

Hydrobiidae

\section{Pyrgophorus coronatum (F.G. Thompson, 1968)}

Archipiélago de San Andrés, Providencia y Santa Catalina: Providencia, Fresh Water, 13 ${ }^{\circ} 20^{\prime} 45.6^{\prime}$ ' N, 81 ${ }^{\circ} 23^{\prime} 21.18^{\prime}$ ', O, entre hojarasca del pozo alto, 40 m, 17 Oct 2013, Daniela Cortés Guzmán (Colecciones zoológicas-ICN 068072); LazyHill- San Felipe, 1321'21.36' N, 81 ${ }^{\circ} 23^{\prime} 17.5^{\prime}$ ' O,

\begin{tabular}{ccccccc} 
& $\mathrm{N}$ & \multicolumn{5}{c}{ Cinv } \\
2 & 3 & Total & 1 & 2 & 3 & Total \\
345 & 69 & 562 & 2.04 & 2.25 & 1.57 & 2.68 \\
1014 & 116 & 2661 & 1.01 & 1.08 & 1.09 & 1.04 \\
102 & 20 & 640 & 1.03 & 1.86 & 1.47 & 1.26 \\
221 & 45 & 269 & 1.80 & 2.52 & 2.61 & 2.58 \\
412 & 158 & 683 & 1.86 & 1.11 & 1.14 & 1.24
\end{tabular}

entre cantos rodados de la parte baja de la quebrada, 31 m, 16 Oct 2013, Daniela Cortés Guzmán (Colecciones zoológicas-ICN 068073); Fresh Water, 13²0'45.6' ' $\mathrm{N}$, 81 23'21.18', O, entre hojarasca del pozo 1, $40 \mathrm{~m}, 27$ Ene 2014, Daniela Cortés Guzmán (Colecciones zoológicas-ICN 068074); Old Town Spring, 13²1'58.74' ' N, $81^{\circ} 22^{\prime} 37.7^{\prime}$ ' O, parte baja de la quebrada, $60 \mathrm{~m}, 15$ Oct 2013, Daniela Cortés Guzmán (Colecciones zoológicasICN 068075); Bailey Spring, 1321' $16.26^{\circ}$ ' N, 81 $21^{\circ}$ '44', O, entre hojarasca del pozo, 38 m, 28 Ene 2014, Daniela Cortés Guzmán (Colecciones zoológicas-ICN 068076); Old Town Spring, 13²1'58.74', N, 81²1'37.68', O, entre hojarasca del pozo 2, 79 m, 28 Ene 2014, Daniela Cortés Guzmán (Colecciones zoológicas-ICN 068077); Old Town Spring, 13²1'58.74', N, 81²2'37.68', O, entre hojarasca del pozo 1, 79 m, 28 Ene 2014, Daniela Cortés Guzmán (Colecciones zoológicas-ICN 068078); El pico, $13^{\circ} 20^{\prime} 2.7^{\prime}$ ' N, 81 $22^{\prime} 9.54^{\prime}$ ' O, entre cantos rodados de la parte baja de la quebrada, $112 \mathrm{~m}, 29$ Ene 2014, Daniela Cortés Guzmán (Colecciones zoológicasICN 068079); Lazy Hill-San Felipe, 13² $1^{\prime} 21.36^{\prime}$ ' $\mathrm{N}$, 81 23 '17.52', O, entre hojarasca del manantial, $31 \mathrm{~m}, 27$ Ene 2014, Daniela Cortés Guzmán (Colecciones zoológicas-ICN 068080); Old Town Spring, 13²1'58.74', N, $81^{\circ} 22^{\prime} 37.68^{\prime}$ ' O, entre hojarasca del pozo 3, $79 \mathrm{~m}, 01$ Ago 20130, Daniela Cortés Guzmán (Colecciones zoológicas-ICN 068081); Lazy Hill-San Felipe, 13²1'21.36', $\mathrm{N}, 81^{\circ} 23^{\prime} 17.52^{\prime}$ ' O, entre cantos rodados de la parte alta de la quebrada, 31 m, 27 Ene 2014, Daniela Cortés Guzmán (Colecciones zoológicas-ICN 068082); Old

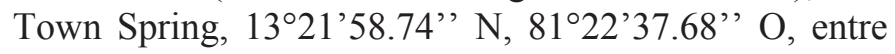
hojarasca del pozo 2, 79 m, 15 Oct 2013, Daniela Cortés Guzmán (Colecciones zoológicas-ICN 068083); Bailey Spring, $13^{\circ} 21^{\prime} 16.26^{\prime}$ ' N, 81 $21^{\prime} 44^{\prime}$ ' O, entre rocas del pozo , 38 m, 28 Ene 2014, Daniela Cortés Guzmán (Colecciones zoológicas-ICN 068084); El Pico, 13²0'2.7', $\mathrm{N}, 81^{\circ} 21^{\prime} 9.54^{\prime} \mathrm{O}$, entre hojarasca de la parte baja de la quebrada , 112 m, 17 Oct 2013, Daniela Cortés Guzmán 
(Colecciones zoológicas-ICN 068085); Lazy Hill-San Felipe, $13^{\circ} 21^{\prime} 21.36^{\prime}$ ' $\mathrm{N}, 81^{\circ} 23^{\prime} 21.36^{\prime} \mathrm{O}$, entre hojarasca de la parte alta de la quebrada , $31 \mathrm{~m}, 16$ Oct 2013, Daniela Cortés Guzmán (Colecciones zoológicas-ICN 068086); Bailey String, $13^{\circ} 21^{\prime} 16.26^{\prime \prime}$ N, $81^{\circ} 21^{\prime} 44$ O, entre raíces del pozo, 38 m, 18 Oct 2013, Daniela Cortés Guzmán (Colecciones zoológicas-ICN 068087); Lazy Hill-San Felipe, $13^{\circ} 21^{\prime} 21.36^{\prime}$ ' N, 81 ${ }^{\circ} 23^{\prime} 17.52$ ' O, entre hojarasca de la parte baja de la quebrada , $31 \mathrm{~m}, 16$ Oct 2013, Daniela Cortés Guzmán (Colecciones zoológicas-ICN 068088); El Pico, 13²0' 2.7' ' N, 81²2'9.54' O, entre cantos rodados de la parte baja de la quebrada , $112 \mathrm{~m}$, 17 Oct 2013, Daniela Cortés Guzmán (Colecciones zoológicas-ICN 068089); Old Town Spring, $13^{\circ} 21^{\prime}$ 58.74', $\mathrm{N}, 81^{\circ} 22^{\prime} 37.68^{\prime} \mathrm{O}$, parte baja de la quebrada , $60 \mathrm{~m}, 15$ Oct 2013, Daniela Cortés Guzmán (Colecciones zoológicas-ICN 068090); Bailey Spring, $13^{\circ} 21^{\prime} 16.26^{\prime}$ ' N, $81^{\circ} 21^{\prime} 44^{\prime} \mathrm{O}$, entre raíces del pozo , 38 m, 28 Ene 2014, Daniela Cortés Guzmán (Colecciones zoológicas-ICN 068091): Bailey Spring, $13^{\circ} 21^{\prime} 16.26^{\prime}$ ' N, 81²1'44' O, entre roca del pozo , 38 m, 18 Oct 2013, Daniela Cortés Guzmán (Colecciones zoológicas-ICN 068092); Lazy Hill-San Felipe, 1321' 21.36' N, 81²3'17.52' O, entre hojarasca de la parte alta de la quebrada , $31 \mathrm{~m}, 27$ Ene del 2014, Daniela Cortés Guzmán (Colecciones zoológicas-ICN 068093); Old Town Spring, $13^{\circ} 21^{\prime} 58.74^{\prime \prime} \mathrm{N}$, $81^{\circ} 22^{\prime} 37.68^{\prime} \mathrm{O}$, entre hojarasca del pozo $1,79 \mathrm{~m}, 15$ Oct 2013, Daniela Cortés Guzmán (Colecciones zoológicasICN 068094); Lazy Hill-San Felipe, 1321' 21.36' N, $81^{\circ} 23^{\prime} 17.52^{\prime} \mathrm{O}$, entre cantos rodados de la parte alta de la quebrada, 31 m,16 Oct 2013, Daniela Cortés Guzmán (Colecciones zoológicas-ICN 068095).

\section{Neritidae}

\section{Neritina punctulata Lamarck, 1816}

Archipiélago de San Andrés, Providencia y Santa Catalina: Providencia, Lazy Hill-San Felipe, 1321'21.36" N, $81^{\circ} 23^{\prime} 17.52$ ' $\mathrm{O}$, en rocas de la parte alta de la quebrada, 31 m, 16 Oct 2013, Daniela Cortés Guzmán (Colecciones zoológicas-ICN 068096); Lazy Hill-San Felipe, $13^{\circ} 21^{\prime} 21.36^{\prime \prime} \mathrm{N}, 81^{\circ} 23^{\prime} 17.52^{\prime}$ ' O, en rocas de la parte alta de la quebrada, 31 m, 29 Jul 2013, Daniela Cortés Guzmán (Colecciones zoológicas-ICN 068097); Lazy Hi11-San Felipe, $13^{\circ} 21^{\prime} 21.36^{\prime \prime}$ N, 81 ${ }^{\circ} 23^{\prime} 17.52$ ' O, en rocas de la parte alta de la quebrada, 31 m, 27 de Ene 2014, Daniela Cortés Guzmán (Colecciones zoológicas-ICN 068098).

\section{Physidae}

\section{Haitia acuta (Draparnaud, 1805)}

Archipiélago de San Andrés, Providencia y Santa Ca- talina: Providencia, Bailey Spring, 1321'16.2', N, $81^{\circ} 21^{\prime} 44^{\prime}$ ' O, entre raíces dentro de un pozo, $38 \mathrm{~m}, 28$ Ene 2014, Daniela Cortés Guzmán (Colecciones zoológicas-ICN 068099); Bailey Spring, $13^{\circ} 21^{\prime} 16.2^{\prime \prime} \mathrm{N}$, $81^{\circ} 21^{\prime} 44^{\prime}$ ' O, entre roca del pozo, $38 \mathrm{~m}, 18$ Oct 2013, Daniela Cortés Guzmán (Colecciones zoológicas-ICN

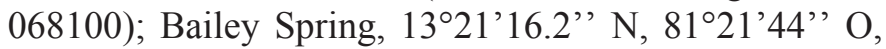
entre raíces del pozo, 38 m, 18 Oct 2013, Daniela Cortés Guzmán (Colecciones zoológicas-ICN 068101); El Pico, $13^{\circ} 20^{\prime} 28.9^{\prime \prime} \mathrm{N}, 81^{\circ} 22^{\prime} 7.2^{\prime \prime} \mathrm{O}$, entre rocas del punto medio de la quebrada, 134 m, 17 Oct 2013, Daniela Cortés Guzmán (Colecciones zoológicas-ICN 068102); El Pico, $13^{\circ} 20^{\prime} 35.7^{\prime}$ ' N, 81 $1^{\circ} 22^{\prime} 7.92^{\prime}$ ' O, entre cantos rodados de la quebrada, 150 m, 17 Oct 2013, Daniela Cortés Guzmán (Colecciones zoológicas-ICN 068103); El Pico, $13^{\circ} 20^{\prime} 2.7^{\prime \prime} \mathrm{N}, 81^{\circ} 22^{\prime} 9.54^{\prime \prime} \mathrm{O}$, entre cantos rodados de la parte baja de la quebrada, 112 m, 29 Ene 2014, Daniela Cortés Guzmán (Colecciones zoológicas-ICN 068104); El Pico, 1320'28.9' ' N, 81 $22^{\circ} 7.2^{\prime}$ ' O, entre roca de la parte media de la quebrada, $131 \mathrm{~m}, 29$ Ene 2014, Daniela Cortés Guzmán (Colecciones zoológicas-ICN 068105); El pico, $13^{\circ} 20^{\prime} 35.7^{\prime \prime} \mathrm{N}, 81^{\circ} 22^{\prime} 7.92^{\prime}$ ' O, entre cantos rodados de la quebrada, 150 m, 29 Ene 2014, Daniela Cortés Guzmán (Colecciones zoológicas-ICN 068106); Lazy

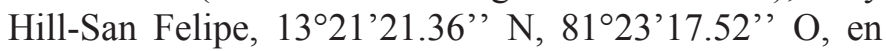
la superficie del manantial, $31 \mathrm{~m}, 16$ Oct 2013, Daniela Cortés Guzmán (Colecciones zoológicas-ICN 068107); Old Town Spring, $13^{\circ} 21^{\prime} 58.74^{\prime}$ ' N, 81 $22^{\circ} 37.68^{\prime}$ ' O, entre hojarasca del pozo 1, 79 m, 28 Ene 2014, Daniela Cortés Guzmán (Colecciones zoológicas-ICN 068108); Lazy Hill-San Felipe, 1321'21.36N, 81²3.37'17.52', $\mathrm{O}$, entre cantos rodados de la parte alta de la quebrada, 31 m, 16 Oct 2013, Daniela Cortés Guzmán (Colecciones zoológicas-ICN 068109); Bailey Spring, 13²1'16.26 $\mathrm{N}, 81^{\circ} 21^{\prime} 44^{\prime \prime} \mathrm{O}$, entre cantos rodados de la parte alta de la quebrada, $31 \mathrm{~m}, 16$ Oct 2013, Daniela Cortés Guzmán (ICN 068110).Old Town Spring, 13²1'58.74 N, $81^{\circ} 22^{\prime} 37.68^{\prime}$ ' O, entre cantos rodados de la parte alta de la quebrada, 31 m, 16 Oct 2013, Daniela Cortés Guzmán (Colecciones zoológicas-ICN 068111).

\section{Planorbidae}

\section{Biomphalaria pallida (Adams, 1846)}

Archipiélago de San Andrés, Providencia y Santa Catalina: Providencia, El Pico, 1320'36.12" N, 81 ${ }^{\circ} 23^{\prime} 7.26^{\prime}$ " $\mathrm{O}$, entre rocas de la parte alta de una quebrada, $161 \mathrm{~m}, 17$ Oct 2013, Daniela Cortés Guzmán (Colecciones zoológicas-ICN 068112); El Pico, 13²0’35.7” N, 81²2'7.92", O, entre cantos rodados de quebrada, 150 m, 29 Ene 2014, Daniela Cortés Guzmán (Colecciones zoológicas-ICN 
068113); El Pico, 13²0’36.12” N, 81²2’7.26’' O, entre superficie de la parte alta de la quebrada, $161 \mathrm{~m}, 29$ Ene 2014, Daniela Cortés Guzmán (Colecciones zoológicas-ICN 068114); Old Town Spring, 1321'58.74” N, $81^{\circ} 22^{\prime} 37.68^{\prime}$ ' O, entre hojarasca del pozo 1, $79 \mathrm{~m}, 28$ Ene 2014, Daniela Cortés Guzmán (Colecciones zoológicas-ICN 068115); Old Town Spring, $13^{\circ} 21^{\prime} 58.74$ " N, $81^{\circ} 22^{\prime} 37.68^{\prime}$ ' O, parte baja de la quebrada, $60 \mathrm{~m}, 15 \mathrm{Oct}$ 2014, Daniela Cortés Guzmán (Colecciones zoológicasICN 068116); El Pico, 13²0’36.12” N, 81²2’7.26”' O, entre la superficie de la parte alta de la quebrada, $161 \mathrm{~m}$, 17 Oct 2013, Daniela Cortés Guzmán (Colecciones zoológicas-ICN 068117); El Pico, 13²0'35.7' N, 81²2'7.92'” O, entre rocas de la quebrada, $161 \mathrm{~m}, 17$ Oct 2013, Daniela Cortés Guzmán (Colecciones zoológicas-ICN 068118); Old Town Spring, $13^{\circ} 21^{\prime} 58.74^{\prime \prime}$ N, 81 ${ }^{\circ} 22^{\prime} 37.68^{\prime \prime}$ O, entre hojas del pozo 1, 79 m, 28 Ene 2014, Daniela Cortés Guzmán (Colecciones zoológicas-ICN 068119). El Pico, $13^{\circ} 20^{\prime} 36.12^{\prime \prime} \mathrm{N}, 81^{\circ} 22^{\prime} 7.26^{\prime}$ ' O, entre cantos rodados de la parte alta de quebrada, $161 \mathrm{~m}, 17$ Oct 2013, Daniela Cortés Guzmán (Colecciones zoológicas-ICN 068120);

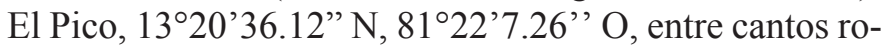
dados de la parte alta de quebrada, $161 \mathrm{~m}, 29$ Oct 2009, Daniela Cortés Guzmán (Colecciones zoológicas-ICN 068121); Bailey Spring, 13²1'16.26” N, 81²1'44” O, entre roca del pozo, 38 m, 18 Oct 2013, Daniela Cortés Guzmán (Colecciones zoológicas-ICN 068122).

\section{DISCUSIÓN}

En la Isla de Providencia, se habían hallado anteriormente cuatro especies dulceacuícolas (G. radiata, Stenophysa marmorata, Truncatella bilabiata y T. caribaeensis; Wurtz 1951), a las que se suman cuatro en este estudio ( $B$. pallida, $H$. acuta, $N$. punctulata y $P$. coronatum), nuevos registros para Providencia y el Archipiélago, de acuerdo a Linares \& Vera (2012).

Del total de macroinvertebrados colectados en el estudio, los moluscos representaron el $4 \%$ de la riqueza y el $29 \%$ de la abundancia, demostrando el importante componente que constituyen en las comunidades de macroinvertebrados dulceacuícolas de la Isla de Providencia (Cortés-Guzmán \& Ospina-Torres 2014).

Al comparar nuestros resultados con los trabajos realizados en otras zonas del Caribe, se encuentran ciertas similitudes. Tres géneros, Biomphalaria, Neritina y Pyrgophorus, se comparten con Nicaragua (Pérez et al. 2008) demostrando la influencia que tiene en la fauna, la zona continental más cercana a la isla. En relación con las islas se encontraron las siguientes similitudes: los géneros Neritina y Pyrgophorus en Tobago (Bass 2003) y en San Cristóbal y Nieves (Bass 2006); dos especies, Neritina punctulata y Physella acuta (sin. Haitia acuta) y el género Biomphallaria en Dominica (Bass 2007); Physa acuta (sin. Haitia acuta) y los géneros Biomphallaria y Pyrgophorus en Cuba (Fimia-Duarte et al. 2010); los géneros Biomphallaria y Pyrgophorus en las Islas Caimán (Bass 2009); N. punctulata en Jamaica (Hyslop \& Hunte-Brown 2012); y Gundlachia radiata. N. punctulata y los géneros Biomphallaria y Pyrgophorus en Martinica (Pointier 2001). Con las islas de Trinidad (Alkins-Koo 1989), Saba (Bass 2008) y San Vicente y Las Granadinas (Bass \& de Silva 2010) la similitud se da apenas a nivel de algunas familias: Planorbidae, Ancylidae e Hydrobiidae en Trinidad; Physidae y Planorbidae en Saba y San Vicente y Las Granadinas.

Es de resaltar la alta abundancia de $P$. coronatum encontrada en este estudio, en relación con el resto de especies encontradas. Taylor (1988) determinó que el género Pyrgophorus tenía su distribución natural restringida a las Islas y al continente alrededor del mar Caribe, y se ha determinado que son especies con potencial invasor y suelen presentarse en altas abundancias (Nava et al. 2011). La evidencia fósil indica que son componentes endógenos de Sudamérica y el Caribe (Wesselingh \& Salo 2006), y no invasoras, sin embargo se ha reportado que tienen una alta tasa de colonización (Nava \& Machado 2013), por lo cual son exitosas colonizando nuevos hábitats, lo que les ha permitido la gran dispersión en la Isla.

La presencia de $B$. pallida, género reconocido por ser transmisor del parásito Schistosoma mansoni, causal de la esquistosomiasis en humanos (DeJong et al. 2001) llama la atención. Esta especie parece estar asociada a ambientes con abundante vegetación, y bajo estas condiciones sus poblaciones pueden alcanzar altas densidades. Un estudio realizado en Cuba por Vásquez-Perera et al. (2010) muestra que $B$. pallida prefiere ambientes naturales, y raramente se observa en ambientes antrópicos. También encontraron que en los ríos, donde hidróbidos y tiáridos suelen habitar, son ambientes preferidos por B. pallida, y que ésta se encuentra bien establecida en ambientes donde suelen ser más comunes otros caracoles de las familias Lymnaeidae, Physidae y Ampullariidae. Fresh Water fue el único sitio donde estuvo ausente esta especie, así como H. acuta. A manera de sugerencia, es posible que la combinación de ambas especies de las familias Hydrobiidae y Physidae ( $P$. coronatum y H. acuta) pudiera favorecer la 
presencia de $B$. pallida en los sistemas de la Isla, aunque se requieren estudios más detallados para confirmar esta observación.

$N$. punctulata sólo estuvo presente en la quebrada de Lazy Hill, ubicada varios metros sobre el nivel del mar (31 msnm), lo que constituye un fenómeno poco observado para esta especie, que suele encontrarse en cercanías a las desembocaduras (Pyron \& Covich 2003). Covich (1988) ha reportado que numerosas especies de gasterópodos y decápodos tropicales colonizan quebradas dulceacuícolas en el Caribe, cerca de la costa marina en las partes bajas, pero son reemplazados por insectos aguas arriba, en tierras más altas, posiblemente por diferencias en las recargas de detritos de hojarasca. Por otro lado, se ha reportado que varios géneros de esta familia (Neritidae) migran estacionalmente río arriba en sus estados juveniles en agrupaciones masivas (Schneider \& Lyons 1993, Pyron \& Covich 2003, Blanco \& Scatena 2005) debido a su ciclo de vida diádromo, y a medida que los organismos crecen se van estableciendo a lo largo del río, con los individuos más grandes en las partes altas de los ríos. Otro estudio en islas, asocia la presencia de especies de este género a la conectividad, permanente o estacional, de las quebradas con el mar (Blanco \& Scatena 2007).

Blanco \& Scatena $(2005,2007)$ han mencionado que los individuos de estos caracoles son significativamente más grandes, pero menos densos en condiciones de flujo más turbulento, y lo contrario en condiciones de menor turbulencia. Blanco-Libreros \& Arroyave-Rincón (2009) encontraron que los caracoles de la familia Neritidae en Puerto Rico se distribuyen a lo largo del río, con los organismos más grandes en las partes altas y los pequeños en las partes bajas, impulsados por el riesgo de depredación de peces moluscívoros en las partes bajas. $N$. punctulata estuvo presente en tres microhábitats de la isla: hojarasca (2 individuos), roca (13 individuos) y cantos rodados (21 individuos). Blanco \& Scatena (2007) observaron que las especies del género Neritina prefieren sustratos de rocas medianas y rugosas, tipos cantos rodados y guijarros, pero principalmente se encuentran en sitios con la combinación de éstos con grava.

$H$. acuta también resultó ser un componente importante, con el $4 \%$ de la abundancia total, y sólo ausente en Fresh Water. Es una especie con una amplia distribución, capaz de vivir en un rango amplio de ambientes dulceacuícolas, con tolerancia a numerosas condiciones ambientales (Turner \& Montgomery 2009). Además es conocida por ser una rápida colonizadora (Chlyeh et al. 2006), por dispersarse fácilmente por el agua y se ha encontrado que puede transportarse en material vegetal en botes entre lagos (Albrecht et al. 2009). Por otro lado, su habilidad de auto-fecundarse promueve la rápida dispersión (Bousset et al. 2004). Gittenberger et al. (2004) observaron que los caracoles adultos de esta especie sobreviven sólo una temporada en la cual dejan una puesta de 18 a 50 huevos, que están rodeados por una mucosidad que les permite adherirse a plantas acuáticas y otras superficies. Dada la dinámica de abundancia que presentó la especie en este estudio, con el mayor valor en la época de lluvias, la dinámica descrita por Gittenberger et al. (2004) se ajusta a nuestros resultados.

En cuanto a las preferencias de microhábitat, nuestros datos concuerdan con el estudio realizado por Harman (1972), en donde se encontró que la presencia y distribución local de los moluscos en hábitats dulceacuícolas está condicionada por los tipos y patrones de sustratos en esos ambientes. Así pues, a mayor cantidad de sustratos disponibles en cierto hábitat, habrá mayor cantidad de moluscos asociados. Los sitios de nuestro muestreo que tuvieron mayor cantidad de microhábitats fueron aquellos donde se encontró la mayor abundancia de moluscos, y un mayor número de especies. Además del tipo, también influyen aquí los patrones del sustrato: aquellos con mayor complejidad permiten que varios grupos de especies ecológicamente similares prosperen sin entrar en contacto entre sí, reduciendo la exclusión por competencia y aumentando la diversidad (Harman 1972).

Como conclusiones, la mayoría de especies encontradas mostraron su mayor abundancia en la época de lluvias, como se esperaba, debido al carácter intermitente de los arroyos estudiados. Adicionalmente el periodo de lluvias presentó la distribución más equitativa de las especies en los sitios. Los microhábitats más sobresalientes fueron los cantos rodados y las raíces. Se encontró que a mayor cantidad de microhábitats disponibles, aumenta la abundancia de los caracoles. Así pues, los sitios con mayor número de microhábitats (Bailey, Old Town y Lazy Hill) tuvieron la mayor abundancia y la mayor diversidad.

La gran abundancia de $P$. coronatum en las quebradas de la isla se debe a su alta capacidad de colonización y a sus características biológicas. La presencia de N. punctulata en la parte alta de un río ha sido poco observada, sin embargo situaciones similares se han reportado para otras especies de la familia. Las dinámicas del ciclo de vida de H. acuta reportadas respaldan los resultados encontrados en este estudio, con un aumento poblacional de la especie en el periodo de lluvias. 
AGRADECIMIENTOS. A la Corporación Coralina por permitirnos el trabajo de campo en la Isla. A la División de Investigación DIB Bogotá de la Universidad Nacional de Colombia por su apoyo financiero para el desarrollo del proyecto. Al departamento de Biología y al Instituto de Ciencias Naturales de la Universidad Nacional de Colombia. Al profesor Rodulfo Ospina por su ayuda en el desarrollo del proyecto.

\section{LITERATURA CITADA}

Aguilera, M. 2010. Geografía económica del Archipiélago de San Andrés, Providencia y Santa Catalina. Documentos de Trabajo sobre Economía Regional No. 133. Centro de Estudios Económicos Regionales (CEER). Banco de la República. Cartagena.

Albrecht C., Kroll, O., Terrazas, E. \& Wilke, T. 2009. Invasion of ancient Lake Titicaca by the globally invasive Physa acuta (Gastropoda: Pulmonata: Hydrophila). Biological invasions, 11: 18211826.

Alkins-Koo, M. A. 1989. The aquatic fauna of two intermittent strea$\mathrm{ms}$ in the southwestern peninsula, Trinidad. Living World, J. Trinidad and Tobago Field Naturalists' Club, 1989-1990: 36-42.

Bacon, P. R. 1978. Notes on Some Freshwater Molluscs from Nariva Swamp, Trinidad. Living World, J. Trinidad and Tobago Field Naturalists' Club, 1978-1979: 14-15.

Baker, H. B. 1924. Land and freshwater mollusks of the Dutch Leeward Islands. Occasional Papers of the Museum of Zoology, University of Michigan, 152: 1-118 + 21 pls.

Bass, D. 2003. A survey of freshwater macroinvertebrates in Tobago. Living World, J. Trinidad and Tobago Field Naturalists' Club, 2003: 64-69.

Bass, D. 2006. A comparison of the Freshwater Macroinvertebrate Assemblages of St. Kitts and Nevis, West Indies. Living World, J. Trinidad and Tobago Field Naturalists' Club, 2006: 30-37.

Bass, D. 2007. Freshwater Macroinvertebrates and Their Habitats in Dominica. Living World, J. Trinidad and Tobago Field Naturalists' Club, 2007: 21-30.

Bass, D. 2008. Freshwater Macroinvertebrates and Their Habitats in Saba, West Indies. Living World, J. Trinidad and Tobago Field Naturalists' Club, 2008: 33-38.

Bass, D. 2009. A Comparison of Macroinvertebrate Communities and Their Freshwater Habitats in the Cayman Islands. Living World, J. Trinidad and Tobago Field Naturalists' Club, 2009: 1-10.

Bass, D. \& de Silva, M. 2010. Invertebrate community structure in a freshwater pond on Mayreau Island, St. Vincent and the Grenadines, West Indies. Living World, J. Trinidad and Tobago Field Naturalists' Club, 2010: 35-42.

Blanco, J. F. \& Scatena, F. N. 2005. Floods, hábitat hydraulics and upstream migration of Neritina virginea (Gastropoda: Neritidae) in Northeastern Puerto Rico. Caribbean Journal of Science, 41: 55-74.

Blanco, J. F. \& Scatena, F. N. 2007. The spatial arragement of Neritina virginea (Gastropoda: Neritidae) during upstream migration in a split-channel reach. River Research and Applications, 23: 235245.

Blanco-Libreros, J. F. \& Arroyave-Rincón, A. 2009. Daños por depredación y tamaño de concha del caracol diádromo Neritina virginea (Gastropoda: Neritidae) en el Río Mameyes, Puerto Rico. Revista de Biología Tropical, 57: 1069-1080.

Bousset, L., Henry, P. Y., Sourrouille, P. \& Jarne, P. 2004. Population biology of the invasive freshwater snail Physa acuta approached throught genetic markers, ecological characterization and demography. Molecular ecology, 13: 2023-2036.

Chlyeh G., Dodet, M., Delay, B., Khallaayoune, K. \& Jarne, P. 2006. Spatio-temporal distribution of freshwater snail species in relation to migration and environmental factors in an irrigated area from Morocco. Hydrobiologia, 553: 129-142.

Cortés-Guzmán, D. \& Ospina-Torres, R. 2014. Comunidades de macroinvertebrados acuáticos en quebradas de la Isla de Providencia. Revista Intrópica, 9: 9-22.

Covich, A. P. 1988. Geographical and historical comparisons of neotropical streams: Biotic diversity and detrital processing in highly variable habitats. Journal of the North American Benthological Society, 7: 361-368.

DeJong, R. J., Morgan, J., Lobato-Paraense, W., Pointier, J. P., Aamarista, M., Ayeh-Kumi, P. F. K., Babiker, A., Barbosa, C. S., Brémond, P., Canese, A. P., Pereira de Souza, C., Domínguez, C., File, S., Gutiérrez, A., Nino-Incani, R., Kawano, T., Kazibwe, F., Kpikpi, J., Lwambo, N. J. S., Mimpfoundi, R., Njiokou, F., Poda, J. N., Sene, M., Velásquez, L. E., Yong, M., Adema, C. M., Hofkin, B. V., Mkoji, G. M. \& Loker, E. S. 2001. Evolutionary relationships and biogeography of Biomphalaria (Gastropoda: Planorbidae) with implications regarding its role as host of the human bloodfluke, Schistosoma mansoni. Molecular biology and evolution, 18: 2225-2239.

Ferguson, F. F. \& Buckmire, K. W. 1974. Notes on the freshwater molluscs of Grenada, British West Indies. Caribbean Journal of Science, 14: 147-148.

Fimia-Duarte, R., Vázquez-Perera, A., Rodríguez, Y. L., Rodríguez, O. C., Pereira-Marín \& C. A., Hernández-Contrera, N. 2010. REDVET Revista electrónica de Veterinaria, 11 (03B): 110.

Gittenberger, E., Janssen, A. W., Kuijper, W. J., Kuiper, J. G. J., Meijer, T., Van der Velde, G. \& de Vries, J. N. 2004. De Nederlandse zoetwatermollusken. Recente en fossiele weekdieren uit zoet en brak water. Nationaal Natuurhistorisch Museum Naturalis, European invertebrate survey-Nederland, KNNV Uitgeverij, Utrecht, The Netherlands.

Gómez, M. I., Strong, E. M. \& Glaubrecht, M. 2011. Redescription and anatomy of the viviparious freshwater gastropod Hemisinus lineolatus (W. Wood, 1828) from Jamaica (Cerithioidea, Thiaridae). Malacologia, 53: 229-250.

Gómez, D. I., Segura-Quintero, C., Sierra-Correa, P. C. \& GarayTinoco, J. (Eds). 2012. Atlas de la Reserva de Biósfera Seaflower. Archipiélago de San Andrés, Providencia y Santa Catalina. Serie de Publicaciones Especiales de INVEMAR No. 28. Instituto de Investigaciones Marinas y Costeras "José Benito Vives De Andréis" -INVEMAR- y Corporación para el Desarrollo Sostenible del Archipiélago de San Andrés, Providencia y Santa Catalina CORALINA. Santa Marta, 180 pp.

Harman, W. N. 1972. Benthic susbstrates: their effect on fresh-water Mollusca. Ecology, 53: 271-277.

Harrison, A. D. \& Rankin, J. J. 1976. Hydrobiological studies of Eastern Lesser Antillean Islands. II. St. Vincent, Freshwater fau- 
na, its distribution, tropical river zonation and biogeography. Archiv für Hydrobiologie, 50: 275-311.

Harrison, A. D. \& Rankin, J. J. 1978. Hydrobiological studies of eastern Lesser Antillean islands, III, St. Vincent; Freshwater Mollusca - their distribution, population dynamics and biology. Archiv für Hydrobiologie, 54: 123-188.

Harrison, A. D. 1984. Redescription of Pyrgophorus parvulus (Gastropoda: Hydrobiidae) from St. Vincent, St. Lucia, and Grenada, West Indies. Proceedings of the Academy of Natural Sciences of Philadelphia, 136: 145-151.

Hyslop, E. J. \& Hunte-Brown, M. 2012. Longitudinal variation in the composition of the benthic macroinvertebrate fauna of a typical North coast Jamaican river. Revista de Biología Tropical, 60: 291-303.

Instituto Geográfico Agustín Codazzi-IGAC. 2008. Atlas Básico de Colombia. Instituto Geográfico Agustín Codazzi. Imprenta Nacional de Colombia. Bogotá.

Lanzer, R. 1996. Ancylidae (Gastropoda: Basommatophora) na América do Sul: Sistemática e distribuicao. Revista Brasileira de Zoologia., 13: 175-210.

Linares, E. L. \& Vera, M. L. 2012. Catálogo de los moluscos continentales de Colombia. Biblioteca José Jerónimo Triana, No. 23. Instituto de Ciencias Naturales, Facultad de Ciencias. Universidad Nacional de Colombia. Bogotá, 360 p.

Merrit, R. W., Cummins, K. W. \& Resh, V. H. 1996. Design of Aquatic insect studies: collecting, sampling and rearing procedures. Pp. 12-28. In: Merrit, R.W. \& K.W. Cummins (Eds.). An introduction to aquatic insects of North America. Kendall/Hunt Publishing, Dubuque.

Nava, M. L. \& Machado, N. E. 2013. Tasa de colonización y estrategia de dispersion de Pyrgophorus coronatum (Caenograstropoda: Hydrobiidae) en una laguna costera, Sistema de Maracaibo, Venezuela. Boletín del Centro de Investigaciones Biológicas, 47: 208-219.

Nava, M., Severeyn, H. \& Machado, N. 2011. Distribución y taxonomía de Pyrgophorus coronatum (Caenogastropoda: Hydrobiidae), en el Sistema de Maracaibo, Venezuela. Revista de Biología Tropical, 59: 1165-1172.

Paraense, W. L. \& Pointier, J.-P. 2003. Physa acuta Draparnaud, 1805 (Gastropoda: Physidae): a study of topotypic specimens. Memórias do Instituto Oswaldo Cruz, Rio de Janeiro, 98: 513-517.

Pérez, A. M., Sotelo, M., Arana, I. \& López, A. 2008. Diversidad de moluscos gasterópodos terrestres en la región del Pacífico de Nicaragua y sus preferencias de hábitat. Revista de Biología Tropical, 56: 317-332.

Pilsbry, H. A. 1920. Review of the Thysanophora group. The Nautilus, 33: 93-96.

Pilsbry, H. A. 1930a. Giffordius pinchoti n. g., n. sp. The Nautilus, 43: 142-143.

Pilsbry, H. A. 1930b. Results of the Pinchot South Sea Expedition. I. Land mollusks of the Caribbean Islands, Grand Cayman, Swan, Old Providence and St. Andrew. Proceedings of the Academy of Natural Sciences of Philadelphia, 82: 221-261, pls. 15-19.

Pointier, J-P. 2001. Invading freshwater snails and biological control in Martinique Island, French West Indies. Memórias do Instituto Oswaldo Cruz, 96: 67-74.

Pyron, M. \& Covich, A. P. 2003. Migration patterns, densities, and growth of Neritina punctulata snails in Rio Espiritu Santo and Rio Mameyes, Northeastern Puerto Rico. Caribbean Journal of Science, 39: 338-347.

Richards, H. G. 1937. Land and freshwater mollusks from the island Cozumel, México, and their bearing on geological history of the región. Proceedings of the American Philosophical Society, 77: 249-262.

Richards, H. G. \& Hummelinck, W. 1940. Land and freshwater mollusks from Margarita island, Venezuela. Notulae Naturae, 62: $1-16$.

Rueda-Delgado, G. 2002. Manual de métodos en limnología. Editorial ACL-Limnos Bogotá, Colombia, 77 pp.

Schneider, D. W. \& Lyons, J. 1993. Dynamics of upstream migration in two species of tropical freshwater snails. Journal of North American Benthological Society, 12: 3-16.

Starmühller, F. \& Therezien, Y. 1982. Résultats de la mission hydrobiologique austro-française de 1979 aux îles de la Guadeloupe, de la Dominique et de la Martinique (Petites Antilles). II-Étude générale de la Dominique et de la Martinique. Revue d'Hydrobiolgie Tropicale, 15: 325-345.

Taylor, D. W. 1988. Aspects of freshwater mollusk ecological biogeography. Palaeogeography, Palaeoclimatology, Palaeoecology, 62: 511-576.

Turner, A. M. \& Montgomery, S. L. 2009. Hydroperiod, predators and the distribution of physid snails across the freshwater habitat gradient. Freshwater biology, 54: 1189-1201.

Van der Schalie, H. 1948. The land and fresh-water mollusks of Puerto Rico. Miscellaneous Publications. University of Michigan. Museum of Zoology, 70: 1-134.

Vázquez, A. A., Sánchez, J. \& Hevia, Y. 2010. Distribution and habitat preferences of the genus Biomphalaria (Gastropoda: Planorbidae) in Cuba. Memórias do Instituto Oswaldo Cruz, 105: 41-44.

Vásquez-Perera, A. A., Sánchez-Noda, J. \& Jiménez, Y. H. 2010. Distribution and habitat preferences of the genus Biomphalaria (Gastropoda: Planorbidae) in Cuba. Memórias do Instituto Oswaldo Cruz, 105: 41-44.

Wantzen, K. M. \& Rueda-Delgado, G. 2009. Técnicas de muestreo de macroinvertebrados bentónicos. In: Domínguez, E. \& H.R. Fernández (Eds.). 2009. Macroinvertebrados bentónicos sudamericanos. Sistemática y biología. Pp. 17-45. Fundación Miguel Lillo, Tucumán, Argentina.

Wege, D. C., Ryan, D., Varty, N., Anadón-Irizarry, V. \& PérezLeroux, A. 2011. Islas del Caribe Hotspot de Biodiversidad. Resumen de Perfil de Ecosistema. Critical Ecosystem Partnership Fund. Conservation International 2011 Crystal Drive, Suite 500 Arlington, VA 22202 USA.

Wesselingh, F. P. \& Salo, J. A. 2006. A Miocene perspective on the evolution of the Amazonian biota. Scripta Geologica, 133: 440458.

Wurtz, C. B. 1950. Results of the Catherwood-Chaplin West Indies Expedition, 1948. Part IV. Land snails of North Cat Cay (Bahamas), Cayo Largo (Cuba), Grand Cayman, Saint Andrews and Old Providence. Proceedings of the Academy of Natural Science of Philadelphia, 102: 95-110.

Wurtz, C. B. 1951. Catalogue of Ancylidae of South and Central America and the West Indies, with description of a new species. The Nautilus, 64: 123-131. 